\title{
BMJ Open Longitudinal survey on the Psychological Impact of the COVID-19 Pandemic in Healthcare Workers (PsyCOVer) in France: study protocol
}

\author{
Leticia Bertuzzi (D) , ${ }^{1}$ Tarik El Aarbaoui, ${ }^{1}$ Mégane Heron, ${ }^{1}$ Anne Gosselin, ${ }^{2,3}$ \\ Laurine Roy-de-Lachaise, ${ }^{1}$ Larissa Fossi, ${ }^{1}$ Francesco Della Corte, ${ }^{4}$ \\ Nicolas Vignier (D) , ${ }^{1,5}$ Maria Melchior (D) , ${ }^{1,3}$ Merritt Schreiber, ${ }^{6}$ \\ Stephanie Vandentorren, ${ }^{7}$ Cécile Vuillermoz (ib ${ }^{1}$
}

To cite: Bertuzzi L, El Aarbaoui T, Heron M, et al. Longitudinal survey on the Psychological Impact of the COVID-19 Pandemic in Healthcare Workers (PsyCOVer) in France: study protocol. BMJ Open 2022;12:e053009. doi:10.1136/ bmjopen-2021-053009

- Prepublication history for this paper is available online. To view these files, please visit the journal online (http://dx.doi. org/10.1136/bmjopen-2021053009).

Received 04 May 2021 Accepted 05 January 2022

Check for updates

(C) Author(s) (or their employer(s)) 2022. Re-use permitted under CC BY-NC. No commercial re-use. See rights and permissions. Published by BMJ.

For numbered affiliations see end of article.

Correspondence to

Dr Leticia Bertuzzi;

lebertuzzi@gmail.com

\section{ABSTRACT}

Introduction In the pandemic, healthcare professionals face even higher levels of stress. It is therefore a priority to estimate the impact of the pandemic on mental health and to propose targeted strategies to improve resilience. The aims of the study were to (1) assess the mental health of healthcare professionals working with patients with COVID-19 and identify social determinants that may increase the risk of negative outcomes; and (2) test the effectiveness of an intervention to improve the resilience of healthcare professionals in France.

Methods and analysis To evaluate the first objective, a national longitudinal study will be carried out among healthcare professionals working with patients with COVID-19. Participants will be recruited via an internet link that will be widely disseminated on social media, mailing lists, medical boards and French medical journals. Primary outcomes are mental health distress/symptoms and resilience. Secondary outcomes are burnout, social and occupational supports and substance use. To meet the second objective, an interventional study will be conducted. The main outcome is the effectiveness of the PsySTART-Responder and the Anticipate.Plan.Deter program. Qualitative analyses will be conducted to understand the strategies used to cope with the pandemic.

Ethics and dissemination The study protocol was approved by the Sorbonne Université Ethical Committee (No 2020-CER-2020-27) and was declared to French Commission on Information Technology and Liberties, CNIL (No2222413, 20-05-2021). The results of this study will provide a better understanding of mental health and social inequalities in mental health among healthcare professionals working in the pandemic; data about the effectiveness of the PsySTART-Responder and the Anticipate.Plan.Deter interventional program in France.

\section{INTRODUCTION}

Healthcare workers are in the frontline of the COVID-19 pandemic. The epidemic occurs in a scenario of pre-existing financial constraints and tensions between health professionals and French policy makers. ${ }^{1}$ The

\section{Strengths and limitations of this study}

- This online longitudinal study will provide knowledge about the dynamic of mental health and social inequalities in mental health among healthcare workers during the COVID-19 pandemic in France.

- The study will assess the effectiveness of the PsySTART-Responder self-report questionnaire and the Anticipate.Plan.Deter interventional program in France.

- This project proposes a methodology that could be easily translated to other disaster contexts and populations.

- The sample will be large and diverse (partnerships with many professional associations) but not random.

- No baseline data from before the COVID-19 pandemic are available to allow comparisons.

deterioration of working conditions and its impact on patient care is one of the reasons for the unprecedented strikes that have been ongoing throughout France since 2019. ${ }^{12}$ In this context of crisis in the health sector, professionals have a higher risk of mental health difficulties such as depression, ${ }^{3-5}$ psychological distress and, in more severe cases, post-traumatic stress disorder (PTSD) ${ }^{6}$

During the SARS-CoV-1 epidemic, a study conducted in Taiwan ${ }^{7}$ indicated a high prevalence of depression $(38.5 \%)$, insomnia (37\%) and PTSD (29.7\%) among healthcare workers. In the beginning of the COVID-19 pandemic, the estimated prevalence of anxiety was $22.0 \%$, and depression was $19.2 \%$ among 214 healthcare workers in Wuhan. ${ }^{8}$ However, a recent meta-analysis about the psychosocial impact of COVID-19 among healthcare workers demonstrated $31.8 \%$ prevalence of depression, $11.4 \%$ 
PTSD, 27.8 insomnia, 46.1\% psychological distress and $37.4 \%$ burnout. $^{9}$ The number of epidemiological studies about physical and mental health significantly increased since the emergence of the COVID-19 pandemic, ${ }^{10}$ and a myriad of studies associate occupational factors with negative mental health outcomes among healthcare professionals.

Professionals involved in the diagnosis, treatment and care of patients infected by the SARS-CoV-2 coronavirus are confronted with increased sources of stress factors. For instance, they may fear for their own life or the life of beloved ones with risks of personal and family contamination (criterion A of the Diagnostic and Statistical Manual of Mental Disorders, Fifth Edition (DSM-5) on post-trauma) ${ }^{11}$; may be exposed to an unusual number of deaths among patients, colleagues or relatives (criterion A of the DSM- 5 on post-trauma $)^{9}$; may have to deal with degraded working conditions ${ }^{12}$ such as exacerbated workload, lack of personal protective equipment and lack of organisation; may have to deal with complex moral and ethical issues ${ }^{13}$ if having to perform expectant triage; may suffer discrimination and stigmatisation for working with patients with COVID- $19^{14}$; and may be exposed to an overabundance of information (infodemic) that might lead to confusion and mistrust. ${ }^{15}$ Therefore, the management of patients with COVID-19 was described as a risk factor for increased anxiety and depression. ${ }^{16-19}$

In addition to the exposed factors, some studies have also suggested the influence of social determinants on negative outcomes. During the SARS-CoV-1 health crisis, a Taiwanese study indicated that psychiatric symptoms were more common among healthcare workers who were young and had little social or family support. ${ }^{7}$ Recent studies show that physicians, nurses, caretakers and other health professionals involved in the management of COVID-19 present higher prevalence of anxiety. ${ }^{9} 2021$ Among nurses, physiotherapists and caretakers, it could be explained by the fact that, besides long-hour shifts, they are in closer contact with patients which can lead to compassion fatigue. ${ }^{20}$ Another study about physicians working with patients with COVID-19 indicated that social support was significantly associated with a good quality of sleep and negatively associated with anxiety and stress. ${ }^{22}$

Regarding media exposure, healthcare workers have not rarely been considered as heroes. If at one hand it adds recognition and improves self-esteem, on the other hand it exacerbates social pressure and, for some, the feeling of illegitimacy. A heroic image goes with the illusion of absence of failure, of illness and the idea that they have to be ready to help at any time. ${ }^{23}$ Nevertheless, in contrast to this heroic status, some healthcare workers have been discriminated and stigmatised due to fear of SARS-CoV-2 exposure. ${ }^{24}$

Healthcare workers often demonstrate difficulties or resistance seeking for help and taking care of themselves. This reluctance is well documented among doctors ${ }^{25} 26$ and could be partially explained by concerns about confidentiality $^{27}$ or by the inadequacy of self-care education. ${ }^{28}$
The French government has been proposing psychosocial support to healthcare professionals since the beginning of the COVID-19 pandemic, mostly by hotlines $(24 / 7)^{29}$ and psychosocial education. To our knowledge, no targeted strategy specific to this population has been offered.

Bearing this in mind, a pilot study was carried out by the French Institute of Health and Medical Research between 18 May and 25 June 2020 to (1) assess the prevalence and factors associated to mental health symptoms in frontline healthcare workers and (2) test the feasibility of the PsySTART-Responder (PsySTART-R) questionnaire and the Anticipate.Plan.Deter (APD) programme to enhance individual capacity to cope with stress factors. PsySTART-R is a self-report questionnaire designed to quickly assess the presence of risk factors to psychological distress. ${ }^{30}$ The APD programme, a stepped care programme, is proposed when individuals are found to have moderate to high levels of stress factors on the PsySTART-R questionnaire with the aim of helping participants create their own resilience plan to cope with stress factors. ${ }^{30}$

Participants were recruited in three different public hospitals in the Paris Region. Forty-two professionals responded to the survey with a satisfactory completion rate (missing data varied between 2\% and 7\%) and a median questionnaire completion time of $18 \mathrm{~min}$. The small proportion of missing data and participants' commitment to the APD programme indicated a good feasibility rate. The small sample size did not allow the significance of the association to be demonstrated; however, preliminary analysis of the responses suggests some results. Almost $60 \%$ of the participants presented signs of depression (measured by Patient Health Questionnaire (PHQ8)) and these symptoms appeared to be more frequent in participants who had been more heavily exposed to stress risk factors according to PsySTART-R (73.3\%) than in those who had been less exposed $(51.8 \%)(\mathrm{p}=0.3026)$. The PTSD score (measured by PTSD Checklist (PCL$5)$ ) was higher among those who reported none or little team support $(24.7)$ than others $(10.9) \quad(p=0.098)$. In contrast, PTSD score was not higher among those who reported little or no support from their hierarchy (10.6) if compared with the others (12.6) $(\mathrm{p}=0.419)$. The results of the pilot study showed good feasibility rates for PsySTART-R and the APD programme in France.

The results from this pilot study raised a need for a more comprehensive study to assess, at a national level, the (1) determinants, particularly social components, that would impact mental health; and (2) to test the effectiveness of PsySTART-R and the APD programme among healthcare professionals rather than those only working in a hospital context. Thus, we propose to conduct a longitudinal epidemiological survey based on the following hypotheses:

1. Healthcare professionals with high levels of exposure to the COVID-19 pandemic (patient care challenges, crisis standards of care, direct life threat, family impact, 
low professional support) may develop mental health symptoms or disorders.

2. There could have been social inequalities in mental health among healthcare professionals due to their distinct social and economic status.

3. Participation in PsySTART-R and the APD programme would lead to reduced symptoms of depression, anxiety and post-traumatic stress, and improved levels of resilience capacity.

4. Mental health disorders might develop in the long term (in particular, anxiety and depression), and a reexposition to COVID-19 surges could deteriorate or worsen the mental health of healthcare workers.

With regard to the previous hypotheses, the PsyCOVer project (Longitudinal survey on the Psychological Impact of the COVID-19 Pandemic in Healthcare Workers) aims to:

1. Assess symptoms of depression, anxiety and posttraumatic stress, as well as resilience capacity among healthcare professionals working with patients with COVID-19 1 year (13-15 months) and 2 years (25-27 months) after the first COVID-19 surge in France (March 2020).

2. Identify social determinants that might influence the mental health of healthcare professionals in the COVID-19 context.

3. Assess the effectiveness of PsySTART-R and the APD program in the pandemic context in France.

4. Assess the resilience strategies used by healthcare workers (APD program) to face the COVID-19 pandemic.

\section{METHODS}

\section{Population study}

We propose to conduct a longitudinal epidemiological survey among healthcare professionals who have been working with patients affected by the COVID-19 in France.

We considered healthcare workers as defined by the French Public Health Code: (1) medical professionals: physicians, midwives, dentists; (2) pharmacists and medical physicists: pharmacists, pharmacy dispensers, hospital pharmacy dispensers, medical physicists; (3) auxiliary paramedics: nurses, nursing assistants, childcare assistant, ambulance driver, physiotherapist, occupational therapist, psychomotrician, medical electroradiology manipulators.

Health-related professions and administrative staff working in health facilities will also be included in the study: (4) embalmer, counsellor, psychologist, social worker; and (5) medical secretary, hospital manager (finance, human resources, information system, logistics, technical, etc), executive or administrative agent (secretary, admissions, archivist, accountant, etc), executive or logistic agent (storekeeper, cook, maintenance staff, etc), manager or technical agent (hospital services agent, stretcher bearer, plumber, heating engineer, electrician, etc).
Participants must be over 18 years, speak one of the study languages (English and French) and give their informed consent. Students or trainees will be eligible to the study.

\section{Recruitment}

The study will be divided into two waves. The first wave will be conducted 13 months from the beginning of the first surge in France (April 2021), and the second wave, 1 year after the first wave of the PsyCOVer project (April 2022). The first wave of the PsyCOVer project has been carried out, by coincidence, in the third and fourth surges of the COVID-19 pandemic in France.

\section{Design study}

First wave

Participants will be recruited via three ways: (1) by an internet link disseminated in social media (Facebook, Twitter and LinkedIn), (2) by an email invitation sent to more than 200 medical and scientific boards (identified a priori) in order to have them disseminate the link of the survey to affiliates/members, and (3) by contacting institutional and academic partners via email, asking for their participation and help in disseminating the internet link.

To participate in the study, healthcare workers will click on the survey link to access the questionnaire and will be asked to answer questions related to inclusion criteria. If inclusion criteria are granted, participants will access the Information Note and will be asked to register by entering their most frequently used email address (either personal or occupational). An email will be automatically sent via the LimeSurvey platform to each participant with a personal link to the questionnaire. As a result of the anonymisation option from the LimeSurvey platform, the email address entered by participants during the registration process will be stored in the 'participant database' and will be separated from the 'questionnaire database'. It will therefore not be possible to link participants' email address to responses.

To access the questionnaire, participants will click on the received personal link. This first page relates to the participants' consent, which will be collected in electronic format on the secure LimeSurvey platform.

\section{Second wave}

All registered participants will be reinvited to participate to the second wave of the study. As in the first wave, participants will receive an email sent directly by the LimeSurvey platform through the 'participant database' in the second wave (April 2022).

\section{Data collection}

The survey will be divided into three parts: the epidemiological part (part 1), the interventional part (part 2) and the evaluation of the interventional part (part 3) (figure 1).

The first wave of the survey (April to June 2021) consists of an epidemiological part to investigate the mental health of healthcare workers (part 1) and an interventional part 


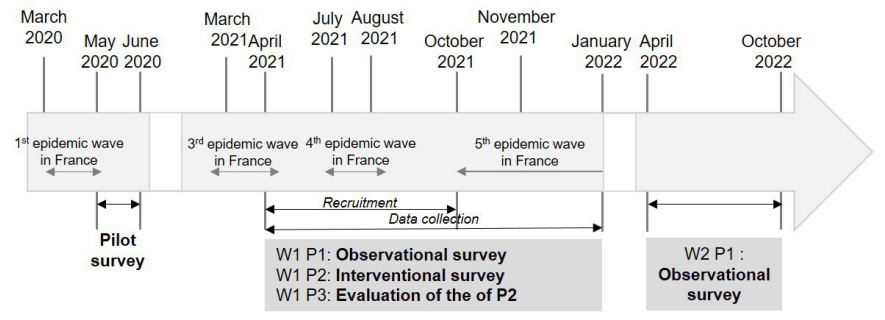

Figure 1 Overview of the Psychological Impact of the COVID-19 Pandemic in Healthcare Workers (PsyCOVer) project. W1 P1, wave 1 part 1; W1 P2, wave 1 part 2; W2 P1, wave 2 part 1; W2 P3, wave 2 part 3.

to assess the efficacy of the PsySTART-R self-report questionnaire and the APD program (part 2).

Parts 1 and 2 will last 6 weeks in total. In week 1 , participants were asked to fulfil parts 1 and 2. In weeks 2, 3 and 4 , the questionnaire will be proposed once a week. In week 6 , information about mental health will be collected again (part 3) to evaluate the efficacy of the intervention (part 2).

The second wave of the survey consists of reconducting the epidemiological survey 1 year after the first wave of the survey.

\section{Patient and public involvement}

Patients or the public were not involved in the design, or conduct, or reporting, or dissemination plans of our research.

\section{Description of the interventional part (part 2)}

The ultimate goal of the intervention in the PsyCOVer project is to increase the participants' ability to cope with stress in this pandemic. First, with the help of the PsySTART-R self-report questionnaire, participants will identify stressors. Second, whenever necessary, they will be proposed to build a personal resilience plan using the APD program.

PsySTART-R COVID- $19^{32}$ is an adapted version of the PsySTART-R questionnaire ${ }^{30}$ that prompts users to indicate which of the 18 risk factors of stress they have experienced during a given period (eg, 24 hours). PsySTART-R does not measure symptoms of acute distress; rather, it measures exposure to the event itself (in this case COVID19-related stressors) including the nature of the event (eg, severe patients), the standard of care (eg, lack of personal protective equipment) and the impact on participants and their families (eg, exposure to virus, life-threatening feeling, unable to return home, concerns about the safety or well-being of relatives).

If participants are exposed to at least one high stress factor or two moderate stress factors, they will be invited to use the self-administered APD program. ${ }^{33}$ APD consists of three different phases:

1. Anticipate: brief introduction to the nature of possible traumatic and cumulative stressors among workers and their impact. Example: the traumatic stress response can include exposure to a life-threatening event, personal or family contamination, witnessing an unusually high number of deaths.

2. Plan: understanding the possible effects of stress on oneself and developing a 'personal resilience plan', which involves identifying and documenting expected stress reactions and response challenges (ie, stressors in specific scenarios, as COVID-19), as well as positive factors such as social support and positive strategies used to face the event and find sense in life. Example: to list expected stress reactions. These can be thoughts, feelings, behaviours and physical symptoms (how do you deal with negative emotions, what do you know about your limits).

3. Determine: implementation of a personal plan developed in the 'Plan' phase. An essential component of this program is learning to monitor one's own exposure to stress so that the user/participant knows when to initiate the personal resilience plan. Example: to monitor reactions to stress and activate the adaptation plan whenever needed.

PsySTART-R and the APD program do not require the presence of trained investigators, something particularly relevant in the context of the COVID-19 pandemic when social distancing is highly recommended.

\section{Measures}

\section{Primary outcomes}

For the present study, primary outcomes for waves 1 and 2 will be (table 1$)$ :

- Depression, assessed by the PHQ-9, ${ }^{34}$ a 9 -item self-reportquestionnaire.

- Generalised anxiety disorder, assessed by the Generalized Anxiety Disorder Scale, ${ }^{35}$ a 7 -item self-report questionnaire.

- PTSD, assessed by the PCL-5, ${ }^{36}$ a 20 -item self-report questionnaire that assesses the 20 DSM- 5 symptoms of PTSD.

- Resilience capacity, assessed by the ConnorDavidson Resilience Scale $10,{ }^{37}$ a 10 -item self-report questionnaire.

- Exposure to stress factors, assessed by the PsySTART-R questionnaire, as described above.

\section{Secondary outcomes}

Burnout is a risk factor of negative mental health outcomes and it has been demonstrated to be prevalent among healthcare workers. ${ }^{38}$ Hence, we propose to investigate its prevalence among healthcare professionals working with patients with COVID-19 in France.

The Maslach Burnout Inventory ${ }^{39}$ is the most commonly used instrument to measure burnout. It captures three dimensions of burnout: emotional exhaustion, depersonalisation and personal accomplishment. For each of the 22 items, the respondent is required to describe his feelings on a 7-point scale.

Social and occupational supports are often mediators of traumatic events and influence the onset of mental health distress/disorders. ${ }^{40}$ These will be investigated separately. 
Table 1 Overview of measures that will be collected in the PsyCOVer project, first and second waves, 2021-2022

Inclusion/first wave

\section{(part 1)}

(Week 1)
Interventional study (first wave, part 2)

(Week 1 to week 4)
Follow-up intervention study (first wave, part 3) (Week 6)

Second wave $\mathrm{X}$

\begin{tabular}{ll}
\hline Information note & $\mathrm{X}$ \\
Institutional Review Board & $\mathrm{X}$
\end{tabular}

Primary outcomes

Depression (PHQ-9)

Anxiety (GAD-7) PTSD (PCL-5)

Resilience capacity (CDRISC-10)

Exposure to stress factors (PsySTART-R)

Secondary outcomes

\begin{tabular}{llll} 
MBI & $X$ & $X$ & $X$ \\
Occupational support & $X$ & $X$ \\
Social support & $X$ & $X$ \\
Substance use & $X$ & & $X$ \\
\hline
\end{tabular}

\section{Other measures}

\begin{tabular}{|c|c|c|}
\hline $\begin{array}{l}\text { Social demographic } \\
\text { characteristics }\end{array}$ & $X$ & $X$ \\
\hline General work-related questions & $X$ & $X$ \\
\hline General health questions & $X$ & $X$ \\
\hline General mental health questions & $X$ & $X$ \\
\hline Perceived discrimination & $X$ & $\mathrm{X}$ \\
\hline Exposure to difficult life events & $X$ & $X$ \\
\hline \multicolumn{3}{|l|}{ Qualitative measure } \\
\hline APD program & & \\
\hline
\end{tabular}

APD, Anticipate.Plan.Deter; CD-RISC-10, Connor-Davidson Resilience Scale; GAD-7, Generalized Anxiety Disorder Scale; MBI, Maslach Burnout Inventory; PCL-5, PTSD Checklist; PHQ-9, Patient Health Questionnaire; PsyCOVer, Psychological Impact of the COVID-19 Pandemic in Healthcare Workers; PsySTART-R, PsySTART-Responder; PTSD, post-traumatic stress disorder.

Occupational and social supports will be assessed using an adapted version of Ruiller's questionnaire. ${ }^{41}$

The consumption of psychoactive substances can be both a cause and a consequence of mental health disorders. We propose to assess it by using an adapted version from the IMPACTS (French acronym for Investigation of post-attacks traumatic events and therapeutic management and support of people involved in the January 2015 terrorist attacks in the Paris Region) Epidemiological Survey. ${ }^{42}$ Tobacco and alcohol consumptions will also be investigated using an adapted version from the same survey.

\section{Other measures}

To identify factors which are potentially associated with mental health outcomes and to test our hypotheses, we will collect (table 1$)$ :
- Sociodemographic characteristics (sex, month and year of birth, level of education, marital status, number of children, income level, country of graduation).

- Work-related questions (profession-specialisation/ training, professional specialty, time in current position at the time of the crisis, year of board inscription/ beginning of clinical practice, change of professional position/role during the crisis, geographic location of exercise-city, type of exercise-individual private practice, multidisciplinary medical centre, public hospital, private hospital).

- Essential questions about health and mental health status (adapted from IMPACTS Epidemiological Survey). ${ }^{42}$

- Perceived discrimination (adapted from IMPACTS Epidemiological Survey). ${ }^{42}$ 
- Exposure to difficult life events (prior to the COVID-19 pandemic) (adapted from IMPACTS Epidemiological Survey). ${ }^{42}$

\section{Qualitative measure}

Participants (who have identified one high stress factor and two moderate factors in PsySTART-R) will be invited to respond to the APD program (table 1). Among the questions, participants will be asked to write down perceived expected stress reactions, perceived response challenges related to stress, a social support plan, as well as positive coping strategies and possible resilience factors. Participants' compliance with PsySTART-R and the APD will be collected.

\section{Sample size and power calculation}

Considering the expected prevalence of the principal outcomes at $14 \%$ for depression, $16 \%$ for anxiety and $20.91 \%$ for PTSD,${ }^{43}$ with a statistic power of $80 \%$ and a value of $5 \%$ for the type I risk, the number of subjects required to have sufficient statistical power is between 415 (for depression), 312 (for anxiety) and 179 (for PTSD). As there is likely that there is $20 \%$ attrition between the two waves of the PsyCOVer survey, we estimated that 519 subjects would be needed in wave 1 to study depression, 390 to study anxiety and 223 to study PTSD. Therefore, 519 subjects will be needed to recruit in the first wave of PsyCOVer project and 415 in the second wave.

\section{ANALYSIS}

In order to answer objectives 1 and 2 (wave 1, part 1), we will conduct descriptive analyses to determine the prevalence of mental health symptoms (primary outcomes) among participants. To test our hypotheses (1 and 2), we will analyse the distribution of these primary outcomes according to participants' sociodemographic characteristics, general work conditions, exposure to stress factors, general health and mental health status, substance use and social support. We will then assess if the primary results indicate that mental health symptoms are more frequent in a specific group and also the presence of social inequalities. In addition to the analyses to address the first objective, we will analyse the distribution of the secondary outcomes (burnout, social and occupational supports and substance use) and identify associated factors to these outcomes. Finally, we will describe the evolution of the outcomes' prevalence between waves 1 and 2. To meet the third objective (wave 1, part 2, and 3 ), we will (1) investigate the relation between the exposures to stress factors measured by PsySTART-R with the primary and secondary outcomes, and (2) compare the evolution of the resilience capacity and mental health symptoms between week 1 and week 6 among participants who used the intervention program compared with those who have not used it.

In the second wave of the survey, a sensitivity analysis will be performed to compare sociodemographic data and mental health status in the first wave for subjects who participated in both waves with those who participate in the first wave of the study but do not participate in the second wave. Finally, to answer the fourth objective, we will perform qualitative analyses on data from the APD program.

For the qualitative analysis, we will consider all the information provided by the participants who engaged in the APD program. First of all, we will list all different responses-expected stress reactions, expected coping strategies (positive and negative) and social support strategies-placing a number next to the response. If multiple participants report the same response, a unique number referring to each of the participants will be listed next to that response. As a result, we will have a list for each APD question and how many participants have mentioned each of the different responses. If two or more respondents refer to the same strategy/plan/action but using different wording, a review team will select and record the wording that they feel is most accurate and most likely to be understood by a member of the target population (healthcare workers). The transcribed data will be coded and analysed using the software program Atlas.ti. ${ }^{44}$

\section{Statistical analysis}

Descriptive analyses regarding the distribution of mental health outcomes will be presented as mean/SD for normally distributed variables, and median/IQR for not normally distributed variables, for continuous data and as proportion for quantitative data. For continuous data, differences between means/medians between groups will be assessed using a t-test for normally distributed or Mann-Whitney-Wilcoxon test for non-normally distributed parameters. Categorical data and differences of proportion between groups will be assessed using a $\chi^{2}$ test or Fisher's test $($ if $n<5)$.

After descriptive analyses, we will identify factors associated to the primary outcomes with regression models and structural equation modelling. Finally, regarding the objective 1 (waves 1 and 2), we will describe the evolution of outcome prevalence in two times: (1) the global trend of change (increasing or decreasing) in prevalence at first and second waves-we will test the significance with McNemar test, and (2) the total change between first and second waves by calculating the proportion of participants who changed from one group to another. ${ }^{45}$ The proportion of change's significance will be tested by calculating the $95 \%$ CI $( \pm 1.96$ times the $\mathrm{SE})$.

Analysis will be carried out in $\mathrm{R}$ version 4.1.0. ${ }^{46}$

\section{Ethics}

The study protocol was approved by the Sorbonne Université Ethical Committee (No 2020-CER-2020-27) and was reported to the French Commission on Information Technology and Liberties, CNIL ( ${ }^{\circ} 2222413$, 20-05-2021). All methods will be performed in accordance with the relevant guidelines and regulations. Participants may contact the research team to discuss the findings of the study 
which will be in the form of aggregated results as data will be anonymous and non-identifiable to the researcher.

The diffusion of study results will be implemented rapidly so that healthcare workers could benefit from it, especially if a new surge of the COVID-19 pandemic occurs. Thus, we will be able to provide the scientific community with knowledge about a population that is not often surveyed with regard to mental health. Besides, we will also be able to present information about the efficacy of a program to monitor levels of exposure to stress factors and to propose the use of a personal resilience plan to mitigate stress.

\section{Study status}

Recruitment started on April 19, 2022 and ended on October 19, 2022. Data collection for the first wave began on April 19, 2022 and ended on January 12, 2022.

\author{
Author affiliations \\ ${ }^{1}$ Social epidemiology research team, Sorbonne Université, INSERM, Institut Pierre \\ Louis d'Epidémiologie et de Santé Publique, IPLESP, Paris, F75012, France \\ ${ }^{2}$ SAGESUD Team, CEPED, Paris, France \\ ${ }^{3}$ French Collaborative Institute On Migrations/CNRS, Aubervilliers, France \\ ${ }^{4}$ CRIMEDIM, Università degli Studi del Piemonte Orientale Amedeo Avogadro Scuola \\ di Medicina, Novara, Italy \\ ${ }^{5}$ Centre d'Investigation Clinique Antilles Guyane, CIC Inserm 1424, Centre \\ Hospitalier de Cayenne, Cayenne, French Guiana \\ ${ }^{6}$ Harbor-UCLA Medical Center/David Geffen School of Medicine at UCLA, The \\ Lundquist Institute, Torrance, California, USA \\ ${ }^{7}$ Direction Scientifique et Internationale, Santé publique France, Saint-Maurice, \\ France
}

Acknowledgements The authors are most grateful to all the study participants for their involvement especially given the very difficult context for them. The authors would like to acknowledge the support from Wissam El-Hage for his contribution to the questionnaire. We would also like to thank the PsySTART-R® and Anticipate. Plan.DeterTM (@Copyright 2021) team for the permission to use the instruments.

Contributors LB designed the study, contributed to the questionnaire conceptualisation and drafted the manuscript. TEA contributed to the study design, questionnaire conceptualisation and implementation, and revised the manuscript. $\mathrm{MH}, \mathrm{AG}$ and MS contributed to the questionnaire conceptualisation and revised the manuscript. LF contributed to the questionnaire conceptualisation. LR-d-L and FDC revised the manuscript. NV, MM and SV supervised the study design, contributed to the questionnaire conceptualisation and revised the manuscript. CV designed the study, contributed to the questionnaire conceptualisation and implementation, and revised the manuscript. All authors reviewed the draft versions of the manuscript, and read and approved the final version.

Funding This project was supported by the Agence Nationale de la Recherche (ANR) grant proposal 'RA-Covid-19'.

Competing interests None declared.

Patient and public involvement Patients and/or the public were not involved in the design, or conduct, or reporting, or dissemination plans of this research.

Patient consent for publication Not required.

Provenance and peer review Not commissioned; externally peer reviewed.

Open access This is an open access article distributed in accordance with the Creative Commons Attribution Non Commercial (CC BY-NC 4.0) license, which permits others to distribute, remix, adapt, build upon this work non-commercially, and license their derivative works on different terms, provided the original work is properly cited, appropriate credit is given, any changes made indicated, and the use is non-commercial. See: http://creativecommons.org/licenses/by-nc/4.0/.

\section{ORCID iDs}

Leticia Bertuzzi http://orcid.org/0000-0002-4401-3589

Nicolas Vignier http://orcid.org/0000-0002-9410-9327
Maria Melchior http://orcid.org/0000-0002-2377-619X

Cécile Vuillermoz http://orcid.org/0000-0001-9292-1939

\section{REFERENCES}

1 Dente K. France's health-care system gripped by strikes. The Lancet 2020;395:103-4.

2 Chrisafis A. French medics warn health service is on brink of collapse. The Guardian 2019.

3 Madsen IEH, Nyberg ST, Magnusson Hanson LL, et al. Job strain as a risk factor for clinical depression: systematic review and metaanalysis with additional individual participant data. Psychol Med 2017;47:1342-56.

4 Virtanen M, Jokela M, Madsen IE, et al. Long working hours and depressive symptoms: systematic review and meta-analysis of published studies and unpublished individual participant data. Scand $J$ Work Environ Health 2018;44:239-50.

5 Vizheh M, Qorbani M, Arzaghi SM, et al. The mental health of healthcare workers in the COVID-19 pandemic: a systematic review. J Diabetes Metab Disord 2020:1967-78.

6 Paz García-Vera M, Sanz J, Gutiérrez S. A systematic review of the literature on posttraumatic stress disorder in victims of terrorist attacks. Psychol Rep 2016;119:328-59.

7 Su T-P, Lien T-C, Yang C-Y, et al. Prevalence of psychiatric morbidity and psychological adaptation of the nurses in a structured SARS caring unit during outbreak: a prospective and periodic assessment study in Taiwan. J Psychiatr Res 2007;41:119-30.

8 Ni MY, Yang L, Leung CMC, et al. Mental health, risk factors, and social media use during the COVID-19 epidemic and Cordon Sanitaire among the community and health professionals in Wuhan, China: cross-sectional survey. JMIR Ment Health 2020;7:e19009.

9 Batra K, Singh TP, Sharma M, et al. Investigating the psychological impact of COVID-19 among healthcare workers: a meta-analysis. Int $J$ Environ Res Public Health 2020;17:9096.

10 Salazar de Pablo G, Vaquerizo-Serrano J, Catalan A, et al. Impact of coronavirus syndromes on physical and mental health of health care workers: systematic review and meta-analysis. J Affect Disord 2020;275:48-57.

11 ed.American Psychiatric Association,, American Psychiatric Association,. Diagnostic and statistical manual of mental disorders: DSM-5. 5th. Washington, D.C: American Psychiatric Association, 2013: 947.

12 Lai J, Ma S, Wang Y, et al. Factors associated with mental health outcomes among health care workers exposed to coronavirus disease 2019. JAMA Netw Open 2020;3:e203976.

13 Arie S. Covid-19: can France's ethical support units help doctors make challenging decisions? BMJ 2020;369:m1291.

14 McKay D, Heisler M, Mishori R, et al. Attacks against health-care personnel must stop, especially as the world fights COVID-19. The Lancet 2020;395:1743-5.

15 WHO. An ad hoc WHO technical consultation managing the COVID-19 infodemic: call for action [Internet], 2020. Available: http:// apps.who.int/iris

16 Liu J, Zhu Q, Fan W, et al. Online mental health survey in a medical college in China during the COVID-19 outbreak. Front Psychiatry 2020;11:459.

17 Elbay RY, Kurtulmuș A, Arpacıoğlu S, et al. Depression, anxiety, stress levels of physicians and associated factors in Covid-19 pandemics. Psychiatry Res 2020;290:113130.

18 Karampelias V, Karonis D, Psaroudi V. The psycho-emotional impact of COVID-19 on surgical staff working in emergency departments. Eur J Trauma Emerg Surg 2020;46:747-9.

19 Chew NWS, Lee GKH, Tan BYQ, et al. A multinational, multicentre study on the psychological outcomes and associated physical symptoms amongst healthcare workers during COVID-19 outbreak. Brain Behav Immun 2020;88:559-65.

20 Santé Publique France. Recensement national des cas de COVID-19 chez les professionnels en établissements de santé. Santé publique France [Internet], 2021. Available: http://www. santepubliquefrance.fr/etudes-et-enquetes/recensement-nationaldes-cas-de-covid-19-chez-les-professionnels-en-etablissementsde-sante\#: :text=Depuis\%20le\%20dernier\%20bilan\%20publi\% C3\%A9,\%C3\%A9tablissements\%20participant\%20\%C3\%A0\% 20l'enqu\%C3\%AAte

21 Chamboredon P, Roman C, Colson S. COVID-19 pandemic in France: health emergency experiences from the field. Int Nurs Rev 2020;67:326-33.

22 Xiao X, Zhu X, Fu S, et al. Psychological impact of healthcare workers in China during COVID-19 pneumonia epidemic: a 
multi-center cross-sectional survey investigation. J Affect Disord 2020;274:405-10.

23 Ornell F, Halpern SC, Kessler FHP, et al. The impact of the COVID-19 pandemic on the mental health of healthcare professionals. Cad Saude Publica 2020;36:e00063520.

24 McKay D, Heisler M, Mishori R, et al. Attacks against health-care personnel must stop, especially as the world fights COVID-19. Lancet 2020;395:1743-5.

25 Tyssen R. Health problems and the use of health services among physicians: a review article with particular emphasis on Norwegian studies. Ind Health 2007;45:599-610.

26 Rosvold EO, Bjertness E. Illness behaviour among Norwegian physicians. Scand J Public Health 2002;30:125-32.

27 Kay M, Mitchell G, Clavarino A, et al. Doctors as patients: a systematic review of doctors' health access and the barriers they experience. Br J Gen Pract 2008;58:501-8.

28 Sanchez-Reilly S, Morrison LJ, Carey E, et al. Caring for oneself to care for others: physicians and their self-care. J Support Oncol 2013;11:75-81.

29 Geoffroy PA, Le Goanvic V, Sabbagh O, et al. Psychological support system for hospital workers during the Covid-19 outbreak: rapid design and implementation of the Covid-Psy Hotline. Front Psychiatry 2020;11:511.

30 Schreiber MD, Yin R, Omaish M, et al. Snapshot from Superstorm sandy: American red cross mental health risk surveillance in lower New York state. Ann Emerg Med 2014;64:59-65.

31 Sylwanowicz L, Schreiber M, Anderson C, et al. Rapid triage of mental health risk in emergency medical workers: findings from Typhoon Haiyan. Disaster Med Public Health Prep 2018;12:19-22.

32 Schreiber M. Field operations manual PsySTART- Responder [Internet], 2020. Available: https://sharedsystems.dhsoha.state.or.us/ DHSForms/Served/le3457b.pdf

33 Schreiber M, Cates DS, Formanski S, et al. Maximizing the resilience of healthcare workers in Multi-hazard events: lessons from the 20142015 Ebola response in Africa. Mil Med 2019;184:114-20.

34 Kroenke K, Spitzer RL, Williams JB. The PHQ-9: validity of a brief depression severity measure. J Gen Intern Med 2001;16:606-13.
35 Spitzer RL, Kroenke K, Williams JBW, et al. A brief measure for assessing generalized anxiety disorder: the GAD-7. Arch Intern Med 2006;166:1092-7.

36 Psychometric properties of the PTSD Checklist for Diagnostic and Statistical Manual of Mental Disorders-Fifth Edition (PCL-5) in veterans': Correction to Bovin et al. Psychol Assess 2016;29:638.

37 Campbell-Sills L, Stein MB. Psychometric analysis and refinement of the Connor-davidson resilience scale (CD-RISC): validation of a 10-item measure of resilience. J Trauma Stress 2007;20:1019-28.

38 Shah K, Chaudhari G, Kamrai D. How Essential Is to Focus on Physician's Health and Burnout in Coronavirus (COVID-19) Pandemic? Cureus 2020.

39 Maslach Cet al. Maslach burnout inventory. 21st ed. Consulting psychologists press, 1986.

40 Jung $\mathrm{H}-\mathrm{S}$, Baek $\mathrm{E}$. A structural equation model analysis of the effects of emotional labor and job stress on depression among nurses with long working hours: focusing on the mediating effects of resilience and social support. Work 2020;66:561-8.

41 Ruiller C. Le soutien social au travail : conceptualisation, mesure et influence sur l'épuisement professionnel et l'implication organisationnelle : l'étude d'un cas hospitalier. 1. Rennes, France: Université Rennes, 2008. https://tel.archives-ouvertes.fr/tel00523398v2

42 Vandentorren S, Pirard P, Sanna A, et al. Healthcare provision and the psychological, somatic and social impact on people involved in the terror attacks in January 2015 in Paris: cohort study. Br J Psychiatry 2018;212:207-14.

43 Cénat JM, Mukunzi JN, Noorishad P-G, et al. A systematic review of mental health programs among populations affected by the Ebola virus disease. J Psychosom Res 2020;131:109966.

44 Scientific Software Development $\mathrm{GmbH}$. Atlas.ti Qualitative Analysis [Internet], 2021. Available: https://atlasti.com/product/what-is-atlasti/

45 Twisk J. Applied longitudinal data analysis for epidemiology: a practical guide. Cambridge University Press, 2013.

$46 \mathrm{R}$ Core Team. A language and environment for statistical computing. [Internet], 2020. Available: www.R-project.org 